\title{
BIFURCATIONS CAUSED BY SAMPLING EFFECTS IN ROBOTIC FORCE CONTROL
}

\author{
Gábor Stépan \\ Department of Applied Mechanics \\ Budapest University of Technology and Economics, H-1521 Budapest \\ stepan@mm.bme.hu \\ László L. Kovács \\ Department of Applied Mechanics \\ Budapest University of Technology and Economics, H-1521 Budapest \\ kovacs@mm.bme.hu \\ József Kövecses \\ Department of Mechanical Engineering \\ McGill University, Montreal, QC, Canada, H2A 2K6 \\ jozsef.kovecses@mcgill.ca
}

Abstract

The empirically developed force control in cases of the robotic polishing and the rehabilitation robots serve as a motivation for the study of the peculiar dynamic behaviour of digital force control. The effect of the sampling times of the digital controllers are studied analytically, and the corresponding stability charts are presented for different gain and mechanical parameters describing also the different sampling frequencies at the force sensors and in the digital control loop. The types of bifurcations are also identified at the stability limits. As one of the practical conclusions, the negative role of differential gain is explained in digital force control.

Keywords: Force controlled robots, differential gain, delayed oscillator

\section{Introduction}

When a robot has to interact with the environment, the control of the contact force between its actuator and the workpiece is often required. Force control tries to maintain prescribed contact force. Apart from the disturbing effects 
of the so-called unmodelled high-frequency dynamics of these systems, basic textbooks often call the attention to the destabilizing digital effects, like sampling (Slotine and $\mathrm{Li}, 1991$ ). At the stability limits presented in the parameter space of the sampling time, control gains and further mechanical parameters, several kinds of bifurcations occur, showing a large variety of nonlinear dynamic behaviour. These analytical results have a central role in understanding the technical phenomena and in forming our physical sense needed during the design of force controlled systems.

The present study has been motivated by two laboratory projects. One is the Rehabilitation Robotics (REHAROB IST-1999-13109) project (Arz et al., 2003) that uses force control during the teaching-in phase of the antispastic physiotherapy of patients suffering from the spastic hemiparesis of the upper limbs (Kovács and Stépán, 2003). The other project is the hybrid position/force controlled Newcastle robot designed for turbine blade polishing (Stépán and Haller, 1995). In both practical applications, peculiar dynamical behaviour and unexpected vibrations occurred referring to several bifurcation phenomena during the experiments.

The present study first describes the basic problems of applied force control, then briefly reviews the motivation of this study. The analytical study of the simplified 1 degree-of-freedom (DoF) mechanical models with different sampling effects at the force sensor and in the control loop is explained in details. The results are presented in the form of stability charts referring also to the types of bifurcations in the system. In the concluding section, qualitative explanation is given why differential gains are avoided in practical force control applications like rehabilitation robotics or robotic polishing.

\section{Applied Force Control}

Several excellent books (like Gorinevsky et al., 1997; Siciliano and Villani, 1999; Natale, 2003) have been published recently on force control of robot manipulators, showing the great demand for understanding and synthesizing experiences in this field. These books investigate models of several degrees of freedom in cases of continuous-time force control. The experiments validating the proposed control algorithms, however, are exclusively carried out using digitally controlled experimental testbeds. Only brief qualitative discussions and over-simplified analytical studies are presented regarding the dynamics of the digitally controlled counterparts of these systems. The major simplification in the analytical studies is, that the analyzed mathematical models are scalar and of first order only, i.e., the inertial forces are often neglected compared to the viscous damping ones. In the meantime, the persistent investigation of the delayed oscillators (Stépán, 1989) call the attention to the difficulty of 
the mathematical problem here, also having important physical consequences, explaining unexpected vibration phenomena.

The reassuring statement, that the digital controller with increasing sampling frequency should approach the characteristics of the corresponding continuous controller, can be posed in a confusing way, too: any finite sampling time results in substantially smaller gain parameters than the corresponding analogue controllers may have. Apart from these general (and not contradictory) statements, it is much less studied that the decrease of sampling frequency does not decrease maximum gains uniformly at the limit of stability, and a large sampling time may result in better stability properties in oscillatory systems than small ones have.

It depends on the control parameters and mechanical properties of the system whether a control algorithm with a certain sampling frequency can be considered continuous, or the digital effects have to be taken into account. For example, a digital force control algorithm with high sampling frequency can be considered "continuous" if the end effector of the robot comes to contact with a soft environment where the effective stiffness of the system is very low and the effective (or modal) mass is high. However, if the environment is very stiff, or there are high-frequency vibration modes with low modal damping, then the effects of the discrete-time nature of the controller will have significant influence on the dynamic behaviour of the force controlled system even at high sampling rate. This significant influence means, for example, that the maximum stable proportional gains are severely limited in these cases.

\section{Motivation}

The stability properties of the 3 degree-of-freedom (DoF) hybrid position/force controlled Newcastle robot was analysed in detail both theoretically and experimentally (Stépán and Haller, 1995). The robot was used and designed for turbine blade polishing, and it maintained constant contact force between the polisher and the blade. The bending stiffness of the polished blade was strongly determined by the principal directions of the matrix of area moment of inertia of the wing-shaped cross section of the blade. Depending on the direction of the force control that was normal to the blade surface, the robot lost stability and started self-excited oscillations with relatively low frequencies in the range of a couple of Hertz. Figure 1 shows the 2 DoF mechanical model of the robot in the force controlled direction representing the elasticity of the force sensor and also that of the contacted environment, which is the turbine blade in this case.

The experimentally confirmed stability chart shows the stable regions in the plane of the sampling time $\tau$ of the digital controller and the proportional gain $P$ for the identified mechanical parameters not presented here. There are two 

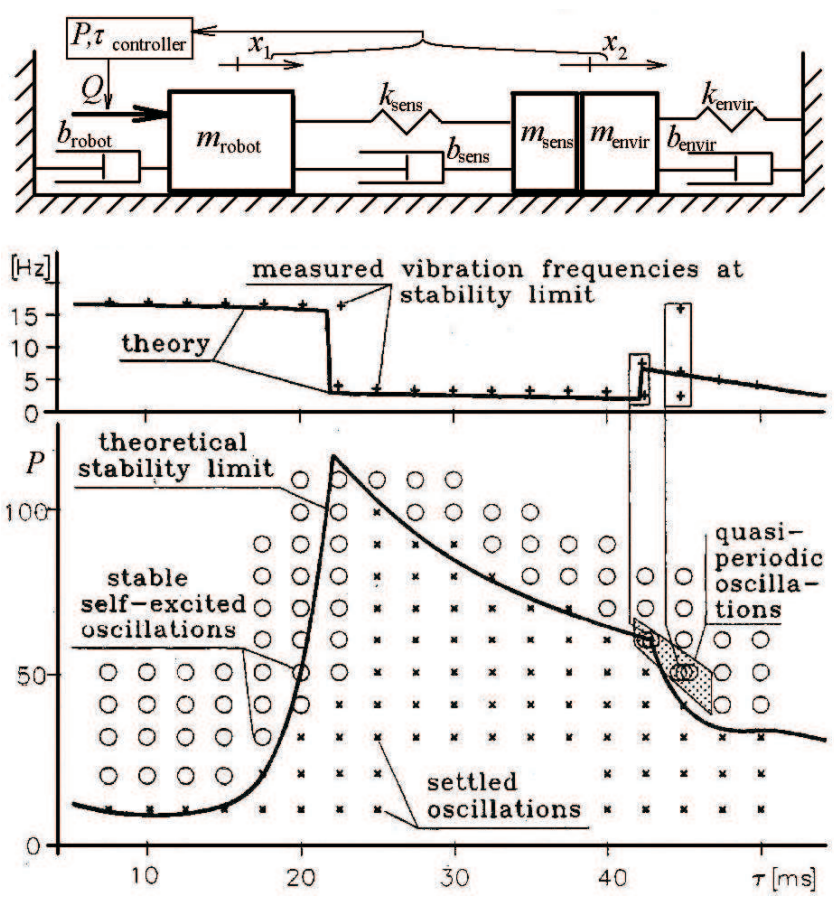

Figure 1. Mechanical model and stability chart for force control in turbine blade polishing

important conclusions of this series of experiments. First, the largest gains can be found not at the lowest realisable sampling times. Second, any attempt to apply the derivative of the force error multiplied with a differential gain $D$ in the digital control resulted in further reduction of the stable domains.

The other example for stability problems with force control comes from the so called RehaRob project (Arz et al., 2003). During the teaching-in phase, the force is controlled between the patient's arm (attached to the orthosis in Fig. 2) and the robotic arm. Figure 2 shows also the corresponding mechanical model and stability chart. The parameter values are not listed here, but the corresponding stability chart shows the stable control parameter region for the real mechanical parameter values. Since the differential gains caused stability problems, again, only proportional gain $P$ and integral gain $I$ were used. Actually, the integral terms did not improve the system behaviour much. In this case, we also experienced the improved stability for large sampling times, but those sampling time values were unreasonably high, so we did not study them further.

All these experiences, and similar reports in the literature directed our investigation to the mechanical root of the problem. In the subsequent sections, we study the simplest possible 1 DoF force control model in case of a PD 


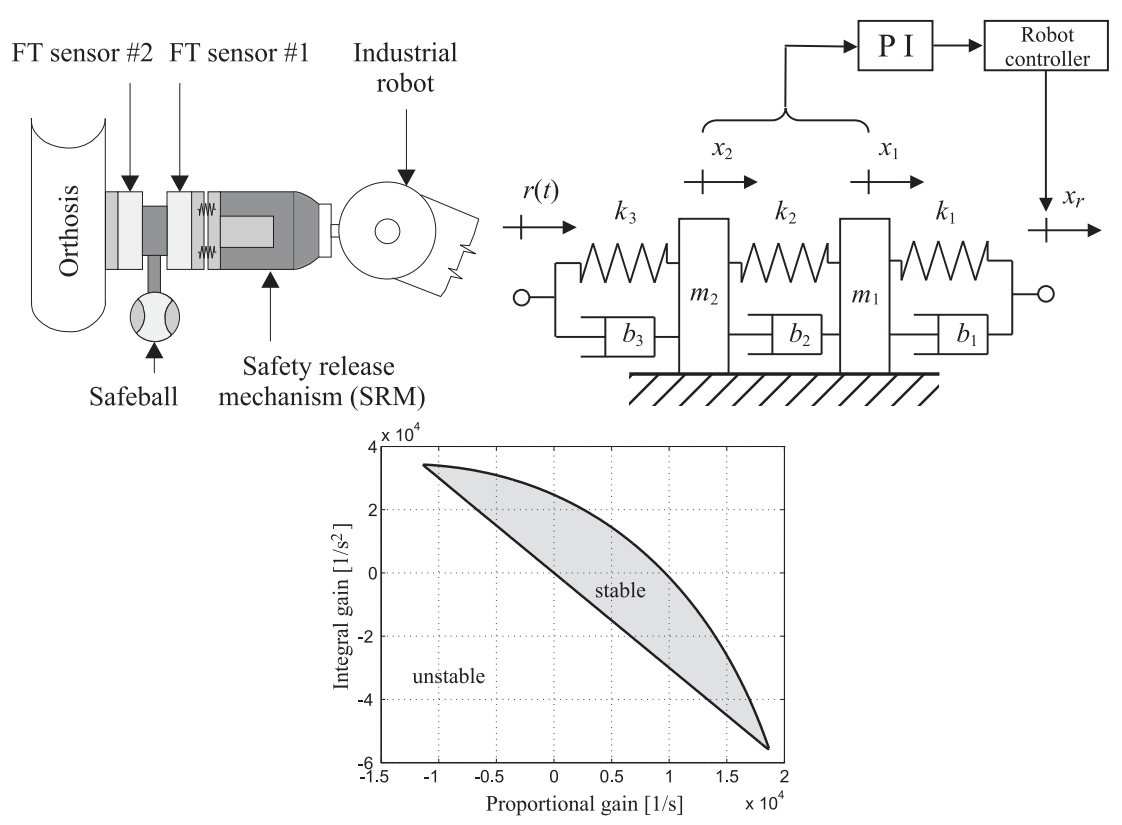

Figure 2. Mechanical model and stability chart for force control applied in rehabilitation robotics

controller with respect to the force error in the presence of the digital effects, namely for finite sampling times.

\section{Mechanical model}

Consider the mechanical model shown in Figure 3. This is a 1 DoF model that can give a good approximation for the behavior of a robotic arm with force control in one direction. The equivalent mass $m$ and equivalent stiffness $k$ represent the inertia and stiffness of the robot and the environment in the force controlled direction. These parameters can either be identified experimentally or calculated using the constraint Jacobian representing the force controlled direction, and the mass and stiffness matrices of the robot (Kövecses et al., 2003). The generalized force $Q$ represents the effects of the joint drives. Similar models are frequently used in other papers and books to analyze force control (Stépán, 2001; Craig, 1986; Gorinevsky et al., 1997; Quian and Schutter, 1992).

In the model presented in Figure 3, the notation $x_{d}$ refers to the position that corresponds to the desired constant force $F_{d}=k x_{d}$, while the coordinate $y=x-x_{d}$ measures the deformation of the spring relative to this desired equilibrium position. The spring is used to represent the interaction force with the environment. Using the force error signal detected via the spring deforma- 


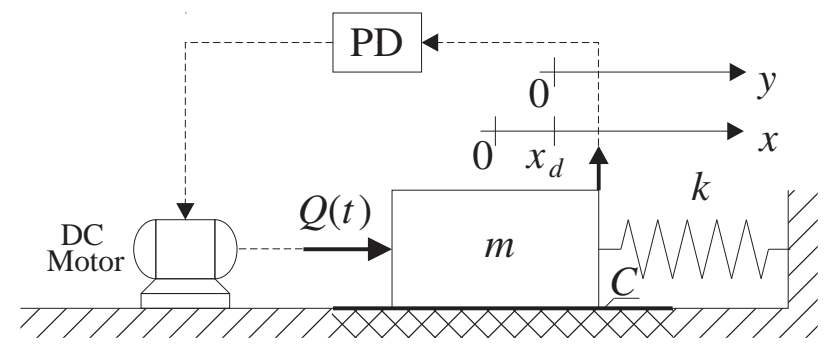

Figure 3. Mechanical model of unidirectional PD force control

tion, the simple PD controller determines the control signal for the DC motor that provides the control force (or torque) $Q$ at the joint drive of the robot. The equation of motion of the above mechanical model can be written as

$$
\begin{aligned}
m \ddot{x}(t) & =-k x(t)-C \operatorname{sgn} \dot{x}(t)+Q(t) \\
Q(t) & =F_{m}(t)-P\left(F_{m}(t)-F_{d}\right)-D \dot{F}_{m}(t)
\end{aligned}
$$

where $P$ and $D$ are the proportional and the differential gains of the PD controller, respectively. In addition, $F_{m}(t)=k x(t)$ denotes the time-dependent measured force. If there is no dry friction considered in the model, i.e. $C \equiv 0$, the trivial solution $x(t) \equiv x_{d}$ satisfies equation (1). In this case, the system can reach the desired equilibrium position $x_{d}$ without a steady state error in principle. Dry friction results in non-zero steady force error, and the higher the proportional gain is, the less this steady force error is (Craig, 1986). This is one of the main reasons why we are interested in applying as large proportional gains as possible, while in the meantime, we often run into stability problems this way. In other words, the steady-state force error cannot be simply eliminated by increasing the proportional gain without the risk of losing stability.

In case of analogue control, however, there is no stability problem to occur in the above simple $1 \mathrm{DoF}$ model. Introducing a perturbation around the desired equilibrium position as

$$
x(t)=x_{d}+y(t),
$$

the equation of motion and its characteristic polynomial $p(\lambda)$ obtained by the exponential trial solution $y(t)=c \exp (\lambda t), c \in \mathbb{R}, \lambda \in \mathbb{C}$ or by Laplace transformation has the form

$$
\begin{aligned}
& m \ddot{y}(t)+D k \dot{y}(t)+P k y(t)=0, \\
& p(\lambda)=\lambda^{2}+D \omega_{\mathrm{n}}^{2} \lambda+P \omega_{\mathrm{n}}^{2}=0
\end{aligned}
$$

where $\omega_{\mathrm{n}}=\sqrt{k / m}$ is the natural angular frequency of the uncontrolled mechanical system. The Routh-Hurwitz criterion yields that the solution $y(t) \equiv 0$ 
corresponding to the desired contact force $F_{m}(t) \equiv F_{d}$, is asymptotically stable for any control gains $P>0, D>0$. There is no upper limit for the gains, which, according to (Craig, 1986), also means that the steady state force error could be eliminated in principle.

However, stable and accurate digital force control requires the analysis of the more refined discrete-time dynamics of the system. As we show in the subsequent sections, this will explain the unexpected bifurcation phenomena for certain proportional gains and sampling frequencies, as well as the undesired destabilising effect of the differential gain.

\section{Digital control model}

To model the digital computer controlled system, we consider a zero-orderhold $(\mathrm{ZOH})$. The force sensor is sampled with the frequency $1 / \tau$, while the digital processor sets the control output at the time instants $t_{n}=n \Delta t$, $n=0,1,2, \ldots$, where $\Delta t$ is the sampling time of the digital control that is considered to be a large integer multiple of the sampling time of the force sensor. Thus, the measured force is available at every sampling instants of the controller, and the time derivative of the measured force can be estimated by finite differences of the measured force values in practice.

The conventional form of the digital control force for a PD controller would be

$$
\begin{array}{r}
Q(t)=(1-P) k y((n-1) \Delta t)-D k \dot{y}((n-1) \Delta t)+k x_{d}, \\
t \in[n \Delta t,(n+1) \Delta t)
\end{array}
$$

and the corresponding equation of motion has the form

$$
\begin{array}{r}
\ddot{y}(t)+\omega_{\mathrm{n}}^{2} y(t)=(1-P) \omega_{\mathrm{n}}^{2} y((n-1) \Delta t)-D \omega_{\mathrm{n}}^{2} \dot{y}((n-1) \Delta t), \\
t \in[n \Delta t,(n+1) \Delta t)
\end{array}
$$

To reduce the number of parameters, introduce the dimensionless time $T=t / \Delta t$, and the notation for the derivatives as

$$
\frac{d}{d T}()=()^{\prime} \text { and } \quad \frac{d}{d t}()=\omega_{\mathrm{n}} \frac{d}{d T}() .
$$

Then the equation of motion is simplified to

$$
y^{\prime \prime}(T)+y(T)=(1-P) y_{n-1}-D \omega_{\mathrm{n}} y_{n-1}^{\prime}, \quad T \in\left[T_{n}, T_{n+1}\right)
$$

To realize digital force control in the presence of the force error derivative, some kind of digital approximation is needed in practice for the time derivative. The simplest possibility is to use a finite difference approximation in the form

$$
\dot{F}_{m}(n \Delta t) \cong \frac{1}{\tau}\left(F_{m}((n-1) \Delta t)-F_{m}((n-1) \Delta t-\tau)\right) .
$$


Now, according to (6), (8) and (7), the equation of motion of the system in the dimensionless time domain can be written in the form

$$
\begin{array}{r}
y^{\prime \prime}(T)+y(T)=(1-P) y_{n-1}-\frac{D \omega_{n}}{(1-q) \Delta T}\left(y_{n-1}-y_{n-2+q}\right) \\
T \in\left[T_{n}, T_{n+1}\right)
\end{array}
$$

where

$$
q=\frac{\Delta t-\tau}{\Delta t}, \quad \Delta T=\omega_{n} \Delta t \quad \text { and } \quad y_{n-2+q}=y((n-2+q) \Delta T)
$$

The new parameter $q$ can be interpreted as a sampling quotient relating the sampling time of the force sensor to the sampling time of the controller. Comparing the resulting equation with (8), we can see that the differential term here is divided by the dimensionless sampling time $\Delta T$. Therefore, we can foresee that for high ratios of the mechanical system natural frequency $f_{n}$ and the control system sampling frequency $f_{s}$, i.e., for $f_{n} / f_{s}=\Delta T /(2 \pi)$, the control will be increasingly similar to the simple proportional controller case when $d=D \omega_{n}=0$.

\section{Stability analysis and bifurcations}

The procedure of the stability analysis of the above equation of motion (10) is based on the construction of a discrete map using the piecewise analytic solution of the non-homogeneous equation of motion for each sampling interval, where the non-homogeneous term is piecewise constant. We need to calculate the position of the robot not only at the sampling instants $T_{n}=n \Delta T$ of the controller, but also at every $T_{n-1+q}=(n-1+q) \Delta T$ instants in order to obtain the measured contact force for the finite difference approximation of the contact force derivative. The lengthy algebraic manipulation results in a simple discrete map in the form

$$
\mathbf{z}_{n+1}=\mathbf{W} \mathbf{z}_{n},
$$

where the 7 dimensional discrete state vector is chosen naturally as

$$
\mathbf{z}_{n}=\left[y_{n}, y_{n}^{\prime}, y_{n-2+q}, y_{n-1}, y_{n-1}^{\prime}, y_{n-3+q}, y_{n-2}\right]^{T}
$$

Using the common robotic notations $\cos (\Delta T)=c_{\Delta T}$ and $\sin (\Delta T)=s_{\Delta T}$, the transition matrix $\mathbf{W}$ of the above mapping can be written in the form 
$\mathbf{W}=\left(\begin{array}{ccccccc}c_{\Delta T} & s_{\Delta T} & \left(1-c_{\Delta T}\right) \tilde{D} & \left(1-c_{\Delta T}\right) \tilde{P} & 0 & 0 & 0 \\ -s_{\Delta T} & c_{\Delta T} & s_{\Delta T} \tilde{D} & s_{\Delta T} \tilde{P} & 0 & 0 & 0 \\ 0 & 0 & 0 & c_{q \Delta T} & s_{q \Delta T} & \left(1-c_{q \Delta T}\right) \tilde{D} & \left(1-c_{q \Delta T}\right) \tilde{P} \\ 1 & 0 & 0 & 0 & 0 & 0 & 0 \\ 0 & 1 & 0 & 0 & 0 & 0 & 0 \\ 0 & 0 & 1 & 0 & 0 & 0 & 0 \\ 0 & 0 & 0 & 1 & 0 & 0 & 0\end{array}\right)$

where

$$
\tilde{D}=\frac{D \omega_{n}}{(1-q) \Delta T} \quad \text { and } \quad \tilde{P}=1-P-\frac{D \omega_{n}}{(1-q) \Delta T}
$$

The exponential stability of the digital force control is equivalent to the stability of the above discrete mapping, i.e., to the convergence of this vector geometric series. Consequently, the stability of the system can be investigated by checking the 7 eigenvalues $\mu_{1, \ldots, 7}$ of the transition matrix $\mathbf{W}$, whether these eigenvalues are located within the open unit disc of the complex plane. With the help of standard numerical methods, we can check this condition of stability.

The corresponding $f_{n} / f_{s}-P$ stability charts are calculated for small $(d=0.1)$ and high $(d=1)$ dimensionless differential gains and presented in Figure 4 and 5, respectively. These figures show the deviations from the ideal charts constructed for zero force sensor sampling time $\tau=0$ as the finite difference approximation of the derivative of the measured force shows up with $\tau>0$. The shaded domain bounded by the thick solid lines refer to the case when the time derivative of the measured force is considered as a continuous input signal of the controller, i.e., the sampling time of the force/torque sensor is negligible compared to the sampling time of the digital force control loop. In this case, the shaded stable domains also illustrate the periodic nature of the $f_{n} / f_{s}-P$ stability charts.

The thin solid line presents the deviations from these charts, when the finite difference approximation is characterized by $\tau=0.1 \Delta t$, i.e. the sampling frequency of the force sensor is ten times higher than the sampling frequency of the force controller. The dashed line shows the case when the measured force is sampled only five times in a sample period of the controller, i.e. $\tau=0.2 \Delta t$.

Figure 4 shows that the finite difference approximation of the measured force causes that the periodic nature of the stability chart disappears with the increase of the frequency ratio. Moreover, the shape of the stable domain of control parameters will converge to the shape of the stability chart obtained for zero differential gain (see Stépán, 2001). Thus, for sufficiently high frequency 
ratios, the PD digital force control with finite difference approximation works as a pure proportional control.

Figure 5 presents the case when the differential gain is large. In this case, the above described convergence of the stable domains is less apparent. In the meantime, the deviations of the stability boundaries that correspond to the finite difference approximation at the differential part of the digital force controller are better illustrated. For low frequency ratios, there is not much change in the stability domain due to the finite difference approximation.

The comparison of the charts in Figures 4 and 5 clearly show, that the increase of the differential gain causes loss of most of the stable regions.

The charts also represent those critical eigenvalues $\mu$ of the transition matrix $\mathbf{W}$ that are of modulus 1 at the limit of stability. This clearly shows that

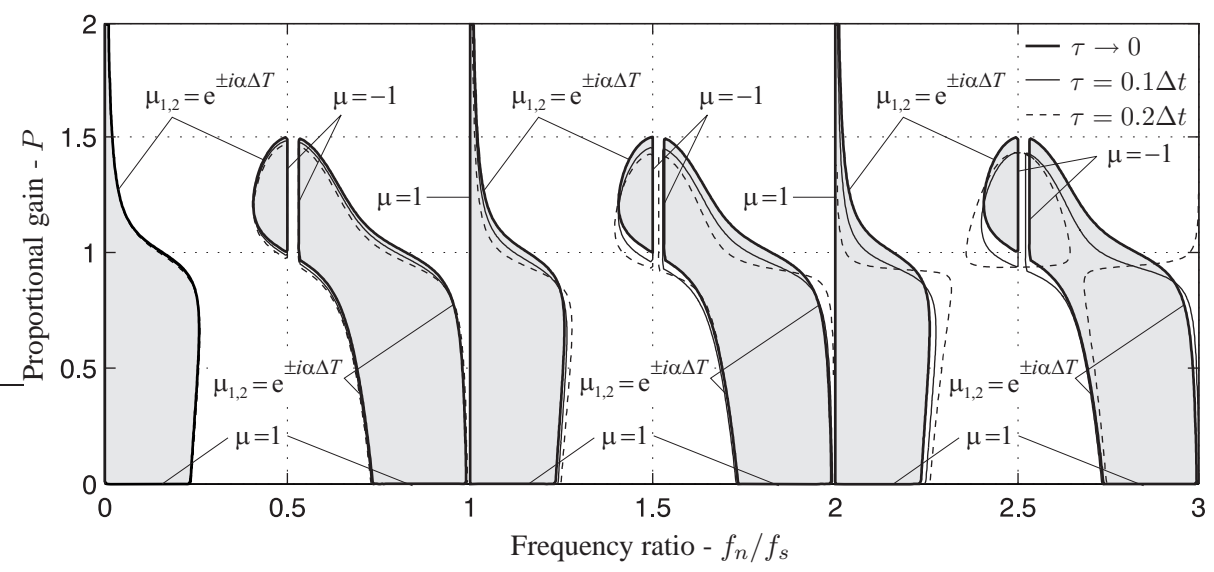

Figure 4. The effect of the finite difference approximation, $d=0.1$

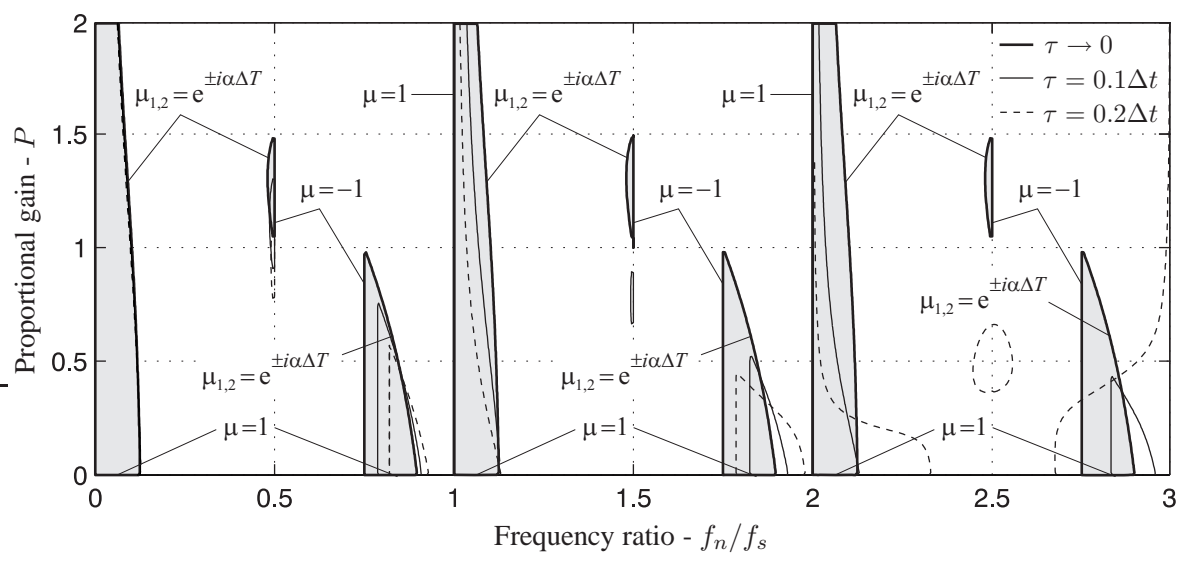

Figure 5. The effect of the finite difference approximation, $d=1$ 
either secondary Hopf (in other words, Neimark-Sacker) bifurcations or period doubling (in other words, flip) bifurcations can occur, corresponding to the complex conjugate pairs of eigenvalues $\mu_{1,2}$, or to the $\mu=-1$ eigenvalue, respectively. This means, that the largest frequency of the arising self-excited vibrations cannot be greater than the half of the sampling frequency of the system.

\section{Conclusion}

The application of differential terms in the digital force control results in the reduction of most of the regions of stability in the parameter space. This effect is the same if a finite difference approximation is used for the force derivative term in the control loop. The finite difference approximation provides a simple kind of filtering of the force derivative signal. For stiff mechanical systems having large natural frequency $f_{n}$, the force control stability properties converge to that of the simple proportional controller.

The stability charts of digital force control show an intricate structure, and several bifurcations can occur at the limits of stability either with or without differential gains in it. The digital effects need special attention during the design of force control in case of stiff mechanical systems with low internal viscous damping to be contacted.

\section{Acknowledgments}

This research was supported by the Hungarian Scientific Research Foundation grant No. T043368, the Natural Sciences and Engineering Research Council of Canada grant No. 206256, and the Bilateral Hungarian-Canadian Society and Technology Program grant No. TéT-BILAT CAN-1/03.

\section{References}

Arz, G., Tóth, A., Fazekas, G., Bratanov, D., and Zlatov, N. (2003). Three-dimensional antispastic physiotherapy with the industrial robots of "Reharob". In Proceedings of The Eight International Conference on Rehabilitation Robotics (ICORR 2003), pages 215-218, Republic of Korea.

Craig, J. J. (1986). Introduction to Robotics Mechanics and Control. Addison-Wesley, Reading, MA.

Gorinevsky, D. M., Formalsky, A. M., and Schneider, A. Yu. (1997). Force Control of Robotics Systems. CRC Press LLC, Boca Raton, Florida.

Kovács, L. L. and Stépán, G. (2003). Dynamics of digital force control applied in rehabilitation robotics. Meccanica, 38(2):213-226.

Kövecses, J., Piedboeuf, J. C., and Lange, C. (2003). Dynamics modeling and simulation of constrained robotic systems. IEEE/ASME Transactions on Mechatronics, 8(2):165-177.

Natale, C. (2003). Interaction Control of Robot Manipulators. Springer-Verlag, Berlin Heidelberg. 
Quian, H. P. and Schutter, J. De (1992). The role of damping and low pass filtering in the stability of discrete time implemented robot force control. In Proceedings of the 1992 IEEE International Conference on Robotics and Automation, pages 1368-1373, Nice, France.

Siciliano, B. and Villani, L. (1999). Robot Force Control. Kluwer Academic Publishers.

Slotine, J. J. E. and Li, W. (1991). Applied Nonlinear Control. Prentice-Hall Inc.

Stépán, G. (1989). Retarded Dynamical Systems. Longman, London.

Stépán, G. (2001). Vibrations of machines subjected to digital force control. International Journal of Solids and Structures, 38:2149-2159.

Stépán, G. and Haller, G. (1995). Quasiperiodic oscillations in robot dynamics. Nonlinear Dynamics, 8:513-528. 